\title{
Beitrag zur Kenntnis der weidenbewohnenden Venturiaceae
}

\section{Doctoral Thesis}

\section{Author(s):}

Nüesch, Jakob

Publication date:

1960

Permanent link:

https://doi.org/10.3929/ethz-a-000088439

Rights / license:

In Copyright - Non-Commercial Use Permitted 
Prom. Nr. 3046 B. DisS ETH

Beitrag

zur Kenntnis der weidenbewohnenden

Venturiaceae

VON DER

EIDGENOSSISCHEN TECHNISCHEN

HOCHSCHULE IN ZÜRICH

ZUR ERLANGUNG

DER WUTDE EINES DOKTORS

DER TECHNISCHEN WISSENSCHAFTEN

GENEHMIGTE

PROMOTIONSARBEIT

VORGELEGT VON

JAKOB N ÚE S CH

DIPL. ING. AGR. ETH

VON BALGACH (ST. GALLEN)

$S_{\odot}$

(3).10kat

出

* $\left(\begin{array}{ll}1 & 4 \\ 4 & 0\end{array}\right.$

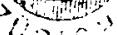

Referent: Herr Prof. Dr. E. GäumanN

Korreferent: Herr Prof. Dr. H. KeRN

1960

Druck von A. W. Hayn's Erben, Berlin West

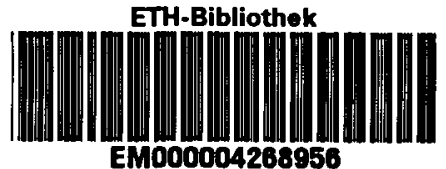


In toten Blättern durchwuchert der Pilz neben der oberflächlichen Ausbreitung der Hyphen das ganze Blattgewebe analog den Venturia-Formen. Auf den lebenden Blättern senkt der Pilz einige haustorienartige Fortsätze in die Epidermis (s. Abb. 11), jedoch treten nie echte nekrotische Realktionen der Wirtszellen auf. Die befallenen Blätter vergilben und fallen frïhzeitig ab. Vermutlich bezieht Epipolaeum longisetosum einen Teil der Nährstoffe aus Blattexsudaten. Auffallend ist bei diesem wenig ausgeprägten Parasitismus die enge Wirtsspezifität wie bei den saprophytischen Formen der Venturia belvetica, Venturia microspora und Venturia subcutanea. Experimentell wurden die beiden Vertreter der Sektion Arbusculoideae Fr., Salix foetida und Salix Waldsteiniana, ferner Salix appendiculata als Wirte nachgewiesen. In der Natur kommt der Pilz auch auf Salix caprea vor (Volkart 1912). Die Verbreitung von Epipolaeum longisetosum ist auf die subalpine und alpine Region beschränkt. Obgleich ihr Wirt Salix caprea auch in tiefen Lagen vorkommt, konnte der Pilz dort bisher nicht gefunden werden.

\section{Auszuschließende Arten}

a) Venturia austro-germanica Rehm

REHM (1907) beschrieb Venturia austro-germanica auf toten Blättern von Salix spec. aus dem Tirol. Müllfr (1955) sammelte in den französischen Alpen auf Salix reticulata einen Pilz, den auch BARR (1959) auf Salix arctica und anderen nicht näher beschriebenen Weiden in Kanada fand. Beide stellten diese Form zu Venturia austro-germanica. BARR (1959) untersuchte die von DeArness (1917) als Venturia subcutanea und später (DeARNess 1923) als Mycosphaerella minor var. reticulata bezeichnete Pilzform von Salix reticulata und identifizierte sie mit Venturia austro-germanica. Auf Grund einer Durchsicht des Typusmateriales von Venturia anstro-germanica aus dem Reichsmuseum Stockholm konnte jedoch festgestellt werden, daß der Wirt falsch bestimmt und nun als Vaccinium myrtillus erkannt wurde.

b) Venturia macrospora Rostr.

Rostrup (1894) beschrieb auf Salix groenlandica auct., einer mit Salix belvetica Vill. verwandten, nordischen Weide Venturia macrospora, welche sich ebenfalls in den Sporendimensionen von Venturia chlorospora unterscheiden soll. Das eingesehene Typusmaterial wies jedoch nur eine Form von Mycosphaerella Tassiana auf; es ist aber denkbar, daß Venturia belvetica auch auf Salix groenlandica vorkommt.

\section{Zusammenfassung}

Auf Grund zahlreicher Kollektionen wurden die weidenbewohnenden Venturiaceae morphologisch untersucht. Ferner konnte an einer größeren Zahl von Isolationen auch ihr Verhalten in Reinkulturen studiert werden. Soweit als möglich wurden mit Hilfe von Infektionsversuchen die Wirtskreise verschiedener Arten umgrenzt. Besonders wurde die Erzielung von Hauptfruchtformen aus Reinkulturen angestrebt. Die erfolgreich angewandten Methoden wurden beschrieben. 
So gelang es, die schon länger diskutierte Beziehung zwischen Venturia chlorospora (Ces.) Karst. einerseits und dem Konidienpilz Pollaccia saliciperda (All. et Tub.) v. ARx anderseits zu klären. Als Hauptfruchtform für Pollaccia saliciperda wurde ein ebenfalls zu Venturia gehörender, aber von Venturia chlorospora verschiedener Ascomycet gefunden und als Venturia saliciperda neu beschrieben.

Unter Berücksichtigung der Ergebnisse aus morphologischen Untersuchungen, Infektions- sowie Kulturversuchen lassen sich die bearbeiteten Pilzformen in sechs verschiedene Arten einteilen:

$\begin{array}{ll}\text { Venturia cblorospora } & \text { Konidienform: Fusicladium } \\ \text { Venturia belvetica } & \text { Konidienform: nicht bekannt } \\ \text { Venturia microspora } & \text { Konidienform: nicht bekannt } \\ \text { Venturia saliciperda } & \text { Konidienform: Pollaccia } \\ \text { Venturia subcutanea } & \text { Konidienform: nicht bekannt } \\ \text { Epipolaeum longisetosum } & \text { Konidienform: nicht bekannt }\end{array}$

\section{Summary}

A large number of Venturiaceae specimens were collected from willows. They were investigated morphologically and their behaviour was studied in vitro. Artificial infections established the host range of the various species. Special stress was laid on the in vitro production of sexual forms; the methods successfully applied to this aim are described.

It was possible to elucidate the recently discussed relation between Venturia chlorospora (Ces.) Karst. and the imperfect fungus Pollaccia saliciperda (All. et Tub.) v. Arx. It was found that the sexual form of Pollaccia saliciperda actually did belong to the genus Venturia. This ascomycete, clearly different from Venturia chlorospora, is described as a new species called Venturia saliciperda.

On the basis of morphological investigations, infections, and in vitro experiments, the fungi studied in this paper can be attributed to six different species:

$\begin{array}{ll}\text { Venturia chlorospora } & \text { asexual form: Fusicladium } \\ \text { Venturia belvetica } & \text { asexual form: unknown } \\ \text { Venturia microspora } & \text { asexual form: unknown } \\ \text { Venturia saliciperda } & \text { asexual form: Pollaccia } \\ \text { Venturia subcutanea } & \text { asexual form: unknown } \\ \text { Epipolaeum longisetosum } & \text { asexual form: unknown }\end{array}$

\section{Literaturverzeichnis}

ADERHOLD, R., 1897: Revision der Species Venturia chlorospora, inaequalis und ditricha autorum. Hedwigia 36, 67-83.

Atcock, N. L., 1924: A dieback and bark disease of willows, attacking the young twigs. Trans. R. Scott. Arboricult. Soc. 38, 128.

- -, 1926: Successional disease in plants as shown in willow rods. Trans. Brit. Mycol. Soc. 11, 161-167.

Allescher, A., und J. N. Schnabl, 1895: Fungi Bavarici Exs. Cent V. Nr. 485. 